\title{
Pulse amplitude and Lempel-Ziv complexity of the cerebrospinal fluid pressure signal
}

\author{
Santamarta $\mathrm{D}^{1}$, Abásolo $\mathrm{D}^{2}$, Fernández $\mathrm{J}^{1}$, Hornero $\mathrm{R}^{3}$ \\ ${ }^{1}$ Department of Neurosurgery, University Hospital of León, Spain. \\ ${ }^{2}$ Centre for Biomedical Engineering, Division of Mechanical, Medical and Aerospace Engineering, FEPS, \\ University of Surrey, United Kingdom. \\ ${ }^{3}$ Department of Signal Theory and Communications, ETSIT, University of Valladolid, Spain.
}

$\begin{array}{ll}\text { Address for correspondence: } & \text { David Santamarta } \\ & \text { Servicio de Neurocirugía } \\ & \text { Hospital Universitario de León } \\ & \text { Altos de Nava, s/n } \\ & 24080 \text { León } \\ & \text { Spain } \\ & \text { Telephone: }+34987237400 \\ & \text { Fax: } \quad+34987240856 \\ & \text { e-mail: dsantamarta@ saludcastillayleon.es }\end{array}$

\section{SUMMARY}

Background: The complexity of the intracranial pressure signal (ICP) decreases with intracranial hypertension in children with acute brain injury as well as during infusion studies in adults with hydrocephalus. In this study we have analyzed the pressure signal obtained in the lumbar subarachnoid space during infusion testing. Pulse amplitude rises when ICP is increased by external volume addition. Our objective was to determine the relative influence of the pressure range and pulse amplitude on the loss of complexity observed during infusion-related intracranial hypertension.

Material and methods: The Lempel-Ziv (LZ) complexity of the cerebrospinal fluid pressure (CSFP) signal was analyzed in 52 infusion studies performed in patients with normal pressure hydrocephalus (median age 71 years, IQR: 60-78). Four sequences during the baseline, infusion, steady plateau and recovery periods of each infusion study were selected. The mean values of the CSFP (mCSFP), pulse amplitude and LZ complexity in every sequence were measured. Correlations between LZ complexity and CSFP parameters were explored. 
Results: Significant inverse correlations were found between LZ complexity, pulse amplitude and mCSFP during all periods of infusion testing, except at baseline. Partial correlation analysis controlling the effect of mCSFP emphasized the relationship between pulse amplitude and LZ complexity. When pulse amplitude is held constant the partial correlation between LZ complexity and mCSFP is not significant.

Conclusions: The pulse amplitude of the CSFP signal seems to be a major determinant of the waveform complexity.

Key words: intracranial pressure, cerebrospinal fluid, pulse pressure waveform, hydrocephalus, LempelZiv complexity, non-linear analysis.

\section{INTRODUCTION}

The metric of complexity proposed by Lempel and Ziv to evaluate the randomness of finite sequences has been widely used in biomedical signal analysis (1). This complexity measure is related to the number of distinct substrings (i.e., patterns) and the rate of their occurrence along a given sequence of symbols (12). During intracranial hypertension the Lempel-Ziv (LZ) complexity of the intracranial pressure (ICP) signal decreases in children with traumatic brain injury (10), as well as in infusion studies performed in adult patients with hydrocephalus (13).

A rise in pulse amplitude occurs when CSF pressure (CSFP) is increased by an external volume addition (3). We hypothesized that the loss of complexity in the CSFP signal during the infusion period mainly depends on changes in pulse amplitude. The goal of this study was to determine the relative influence of mean CSFP and pulse amplitude on the loss of complexity observed during the infusion period.

\section{MATERIAL AND METHODS}

We analysed retrospectively 52 infusion studies performed in the Department of Neurosurgery, University Hospital of León, Spain. All patients presented clinical symptoms of hydrocephalus and ventriculomegaly. The ventricular size was measured with the Evans ratio. Measurement of CSF outflow resistance $\left(\mathrm{R}_{\mathrm{OUT}}\right)$ through a constant-rate lumbar infusion study is part of the usual clinical routine in the management of patients with chronically developed hydrocephalus. Complexity analysis of the CSFP 
signal was made off line. The local ethics committee approved the study. Informed consent was obtained from either the patient or a close relative.

\section{Lumbar infusion test and derived parameters}

The infusion studies were performed using a variant of the original method described by Katzman and Hussey (11). The procedure has been previously described in detail (13) and will only be summarized here. With the patient in the lateral recumbent position, two lumbar needles (19 Gauge) were inserted in the lower lumbar region. The rostral cannula was connected via a three-way stopcock equipped with a short extension line to a pressure microtransducer $\left(\right.$ Codman ${ }^{\circledR}$ MicroSensor ${ }^{\mathrm{TM}}$ ICP transducer, Codman \& Shurtleff, Raynham, MA). Through the caudal needle, Ringer solution was infused at a constant rate of $1.5 \mathrm{ml} / \mathrm{min}$. The infusion was switched off when a steady plateau pressure level was achieved.

For every infusion study, we carefully selected four artefact-free time windows during the baseline $\left(\mathrm{W}_{0}\right)$, early infusion $\left(\mathrm{W}_{1}\right)$, steady state plateau $\left(\mathrm{W}_{2}\right)$ and the recovery $\left(\mathrm{W}_{3}\right)$ segments of the infusion studies. Mean values of CSFP, pulse amplitude and LZ complexity of the four sequences were determined in every infusion study. Amplitude was defined as the peak-to-peak value of the pulse wave. $R_{\text {OUt }}$ was calculated as the plateau pressure minus opening pressure, divided by the infusion rate.

\section{Data acquisition and complexity analysis}

The pressure signal from the analogue output of the monitor was amplified and digitized (PowerLab 2/25 Data recording system ML825, ADI Instruments, Milford, MA). The analogue-to-digital converter was fitted into a computer for real-time display, storage and processing of the digital signals (PowerLab software; ADI Instruments, Milford, MA). Pressure data were sampled at a frequency of $100 \mathrm{~Hz}$. LZ complexity analysis was applied to a binary sequence generated from the original signal using the median of a 5 seconds (s) moving window as the threshold. Previous studies have shown that 0-1 conversion is adequate to estimate the LZ complexity in biomedical signals $(14,15)$. There was 4 s. of overlapping between consecutive windows. To compute LZ complexity, the sequence has to be scanned from left to right and a complexity counter is increased by one unit every time a new subsequence of consecutive characters is encountered. The detailed algorithm to estimate LZ complexity can be found elsewhere $(1,14,15)$. In order to obtain a complexity measure which is independent of the sequence length, the complexity value should be normalized. The normalized LZ complexity reflects the arising rate of 
new patterns in the sequence. A larger value of LZ complexity means that the chance of new pattern generation is greater, so the sequence is more complex. The complexity value of a given sequence was normalized with regard to the baseline LZ complexity (LZ0) in order to quantify the dynamics of LZ complexity throughout the infusion study $(\Delta \mathrm{LZn}=\mathrm{LZn} / \mathrm{LZ} 0)$.

\section{Statistical analysis}

Data were analysed using SPSS, version 17.0 (SPSS Inc., Chicago, IL). Quantitative variables were expressed as median and interquartile range (IQR). Association between quantitative variables was assessed by the Spearman rank correlation test. Partial correlation analyses between two variables were performed to control the effect of a third variable. The chosen level of significance was $\mathrm{p}<0.05$.

\section{RESULTS}

Baseline characteristics of patients and the results of the measurements of the CSF parameters to obtain $\mathrm{R}_{\text {OUT }}$ are given in table 1 . LZ complexity values are higher at baseline. During the infusion the complexity of the CSFP signal usually diminishes yielding the lowest value during the steady state plateau (LZ2). $\Delta \mathrm{LZ2}$ values lower than 1 in 44 patients $(85 \%)$ reflect this behaviour.

Table 2 summarizes LZ complexity values along with pulse amplitude and mean CSFP in the four segments of the infusion tests. As expected, the amplitude of the wave increases during the infusion. All parameters tend to recover towards baseline level once the infusion has been interrupted.

The results of correlation analysis between LZ complexity, pulse amplitude and mean CSFP revealed significant inverse correlations during all periods of the infusion testing, except baseline (figure 1). The correlation coefficients were always higher between $\mathrm{LZ}$ complexity and pulse amplitude $\left(\mathrm{W}_{1}\right.$ : rho= -0.82 , $p<0.01 ; \mathrm{W}_{2}:$ rho $=-0.83, p<0.01 ; \mathrm{W}_{3}:$ rho=-0.76, $\left.p<0.01\right)$ than between LZ complexity and mean CSF pressure $\left(\mathrm{W}_{1}:\right.$ rho $=-0.54, p<0.01 ; \mathrm{W}_{2}: \mathrm{rho}=-0.63, p<0.01 ; \mathrm{W}_{3}:$ rho $\left.=-0.41, p<0.01\right)$.

Partial correlations were performed given the known dependence between mean CSFP and amplitude during infusion studies. A first-order partial correlation holding constant the effect of mean CSFP confirms the correlation between LZ complexity and pulse amplitude during the infusion $\left(\mathrm{W}_{1}:\right.$ rho=- 0.40 , $p<0.01 ; \mathrm{W}_{2}$ : rho=-0.32, $\left.p<0.05\right)$ and during the recovery period $\left(\mathrm{W}_{3}:\right.$ rho=-0.38, $\left.p<0.01\right)$. However, the 
partial correlation coefficient controlling the effect of the amplitude rejects a correlation between LZ complexity and mean $\operatorname{CSFP}\left(\mathrm{W}_{1}: \mathrm{rho}=-0.18 ; p=0.20 ; \mathrm{W}_{2}:\right.$ rho=-0.23; $p=0.09 ; \mathrm{W}_{3}$ : rho=-0.03; $\left.p=0.82\right)$.

Finally, the magnitude of the complexity change (i.e. $\Delta \mathrm{LZ}$ values) appeared to be inversely correlated with $\mathrm{R}_{\mathrm{OUT}}(\Delta \mathrm{LZ1}$ : rho $=-0.42, p<0.01 ; \Delta \mathrm{LZ2}$ : rho=-0.47, $p<0.01 ; \Delta \mathrm{LZ3}$ : rho=-0.55, $p<0.01)$. These results mean that a high loss of complexity of the CSFP signal (i.e. decomplexification phenomenon) is associated with higher values of $\mathrm{R}_{\text {OUT }}$. No correlations were found between age or ventricular size with $\Delta \mathrm{LZ}$ values.

\section{DISCUSSION}

Most physiological systems behave non-linearly. They display irregular variations without being random. A major feature of non-linear phenomena is the sensitivity of the generating system to variations in the initial conditions. Non-linear dynamics studies this system's feature as one cause of complex behaviour. Physiological variability in ICP dynamics has been acknowledged since the early period of ICP monitoring $(4,8)$. Thorough analysis of the CSF pulse wave through visual inspection of printed recordings was enough to recognize the spontaneous variations in all components of the wave, as well as the changes in the wave configuration under specific conditions $(4,7,9)$. At that time, it was thought that the wave morphology could have potentially important clinical implications. This assumption, however, was pushed aside until recently, when renewed interest in the pulsatile component of ICP dynamics has brought the waveform into the field of discussion once again $(2,5,6)$.

Complex systems analysis provides tools to address this physiological variability. There are a number of approaches to characterize complexity. Lempel and Ziv proposed a useful complexity measure which can characterize the degree of predictability in a time series and that does not require long data segments to compute (12). The signal must be transformed into a finite symbol sequence, which in the context of biomedical signal analysis is usually a binary sequence. LZ complexity analysis is applied to a binary sequence generated from the original signal using a simple threshold. This metric operates like a sliding window algorithm scrutinizing the signal. It deals with the wave morphology and it focuses on the pulsatile component of the pressure signal. Lempel-Ziv algorithm gives the number of distinct patterns contained in the given finite sequence. After normalisation, the relative LZ complexity measure reflects the rate of new pattern occurrences in the investigated sequence. Values of LZ complexity close to 0 correspond to periodic signals, while a value close to 1 corresponds to random noise. 
Hornero et al. have shown in paediatric patients with traumatic brain injury that the LZ complexity of the ICP signal recorded intracranially decreases during periods of acute intracranial hypertension (10). The loss of complexity has also been detected during infusion studies performed in adult patients with hydrocephalus when the CSFP signal is in the range of intracranial hypertension (13).

The main observation from this study is that the loss of complexity in the CSFP signal observed during infusion-related intracranial hypertension seems to be more correlated with the pulse amplitude than with the level of pressure. The correlation coefficients between LZ complexity and pulse amplitude were higher than between LZ complexity and mean CSF pressure throughout all the infusion segments. A partial correlation analysis was performed given the strong correlation between pulse amplitude and the pressure level during infusion testing. The partial correlation coefficient between LZ complexity and pulse amplitude diminishes, but it still remains significant when the effect of mCSFP is controlled. This means that the relationship between both variables is modulated by the variable submitted to control (namely, mean CSFP). The partial correlation coefficient between LZ complexity and mCSFP does not reach statistical significance when controlling the effect of amplitude. This means that the simple inverse correlation between both variables is substantially modified if the effect of the amplitude is held constant. Therefore, we assume that pulse amplitude is a major determinant of CSFP signal complexity. Whether ICP complexity analysis holds relevant clinical information or not has yet to be investigated.

\section{REFERENCES}

1. Aboy M, Hornero R, Abásolo D, Álvarez D (2006) Interpretation of the Lempel-Ziv complexity measure in the context of biomedical signal analysis. IEEE Trans Biomed Eng 53: 2282-2288

2. Anile C, De Bonis P, Albanese A, Di Chirico A, Mangiola A, Petrella G, Santini P (2010) Selection of patients with idiopathic normal-pressure hydrocephalus for shunt placement: a single-institution experience. J Neurosurg 113: 64-73

3. Avezaat CJ, van Eijndhoven JH, Wyper DJ (1979) Cerebrospinal fluid pulse pressure in intracranial volume-pressure relationships. J Neurol Neurosurg Psyquiatry 42: 687-700

4. Cardoso ER, Rowan JO, Galbraith S (1983) Analysis of the cerebrospinal fluid pulse wave in intracranial pressure. J Neurosurg 59: 817-821

5. Carrera E, Kim D-J, Castellani G, Zweifel C, Czosnyka Z, Kasparowicz M, Smielewski P, Pickard JD, Czosnyka M (2010) What shapes pulse amplitude of intracranial pressure ? J Neurotrauma 27: 317-324 
6. Eide PK, Sorteberg W (2010) Diagnostic intracranial pressure monitoring and surgical management in idiopathic normal pressure hydrocephalus: a 6-year review of 214 patients. Neurosurgery 66: 80-91

7. Foltz EL, Aine C (1981) Diagnosis of hydrocephalus by CSF pulse-wave analysis: a clinical study. Surg Neurol 15: 283-293

8. Gega A, Utsumi S, Lida Y, Lida N, Tsuneda S (1980) Analysis of the wave pattern of CSF pulse wave. In: Shulman K; Marmarou A, Miller JD, Becker DP, Hochwald GM, Brock M (eds) Intracranial pressure IV. Springer-Verlag, Berlin/Heidelberg/New York, pp 188-190

9. Hirai O, Handa H, Ishikawa M, Kim S-H (1984) Epidural pulse waveform as an indicator of intracranial pressure dynamics. Surg Neurol 21: 67-74

10. Hornero R, Aboy M, Abasolo D (2007) Analysis of intracranial pressure during intracranial hypertension using Lempel-Ziv complexity: further evidence. Med Bio Eng Comput 45: 617-620

11. Katzman R, Hussey F (1970) A simple constant-infusion manometric test for measurement of CSF absorption. I. Rationale and method. Neurology 20: 534-544.

12. Lempel A, Ziv J (1976) On the complexity of finite sequences. IEEE Trans Inform Theory 22:75-81

13. Santamarta D, Hornero R, Abásolo D, Martínez-Madrigal M, Fernández J, García-Cosamalón J (2010) Complexity analysis of the cerebrospinal fluid pulse waveform during infusion studies. Child's Nerv Syst, published online August 3, 2010; DOI: 10.1007/s00381-010-1244-5

14. Zhang XS, Zhu YS, Thakor NV, Wang ZZ (1999) Detecting ventricular tachycardia and fibrillation by complexity measure. IEEE Trans Biomed Eng 46: 548-555

15. Zhang XS, Roy RJ, Jensen EW (2001) EEG complexity as a measure of depth of anesthesia for patients. IEEE Trans Biomed Eng 48: 1424-1433

\section{CONFLICT OF INTEREST STATEMENT}

The authors declare that they have no conflict of interest.

\section{ACKNOWLEDGMENTS}

This study was supported in part by Consejería de Sanidad, Junta de Castilla y León, project code GRS 493/B/10 given to David Santamarta. 


\begin{tabular}{lc}
\hline \multicolumn{1}{c}{ Characteristic } & Value \\
\hline \hline Sex (No.) & 23 \\
Male & 29 \\
Female & \\
Age: median (IQR) & $71(60-78)$ \\
Years & \\
Etiology (No.) & 23 \\
Idiopathic normal pressure hydrocephalus & 25 \\
Secondary normal pressure hydrocephalus & 4 \\
Shunt dysfunction & \\
Ventricular size: median (IQR) & $0.37(0.35-0.40)$ \\
Evans index & \\
CSF pressure parameters: median (IQR) & $8.21(6.34-11.25)$ \\
Opening pressure (mm Hg) & $26.22(21.14-32.04)$ \\
Plateau pressure (mm Hg) & $12(8.75-15.20)$ \\
CSF outflow resistance (mmHg ml-1 min) & \\
\hline \hline
\end{tabular}

TABLE 1. Baseline characteristics of the patients and CSF parameters during the infusion studies. No., number; IQR, interquartile range.

\begin{tabular}{|c|c|c|c|c|}
\hline & Baseline $\left(W_{0}\right)$ & Infusion $\left(W_{l}\right)$ & Plateau $\left(W_{2}\right)$ & Recovery $\left(W_{3}\right)$ \\
\hline Sequence length $(s)$ & $180(145-200)$ & $300(300-360)$ & $490(455-600)$ & $180(180-240)$ \\
\hline$m C S F P(m m ~ H g)$ & $8.2(6.3-11.2)$ & $17.3(15.1-20)$ & $26.2(21.1-32)$ & $16.8(13.7-19.8)$ \\
\hline$A M P(m m H g)$ & $2.6(1.6-3.4)$ & $5.5(3.5-8.6)$ & $9.7(5.5-13.8)$ & $5.7(3.9-7.6)$ \\
\hline LZ complexity & $0.27(0.24-0.31)$ & $0.21(0.18-0.25)$ & $0.18(0.16-0.24)$ & $0.20(0.18-0.27)$ \\
\hline$\Delta L Z$ & NA & $0.77(0.64-0.95)$ & $0.68(0.57-0.91)$ & $0.75(0.63-0.98)$ \\
\hline
\end{tabular}

TABLE 2. Parameters derived from the cerebrospinal fluid pressure (CSFP) signal in the different periods of the infusion study. Data are presented as median (interquartile range).

s, seconds; mCSFP, mean CSFP; AMP, pulse amplitude; LZ, Lempel-Ziv; $\triangle L Z$, normalized LZ complexity value; NA, not applicable. 

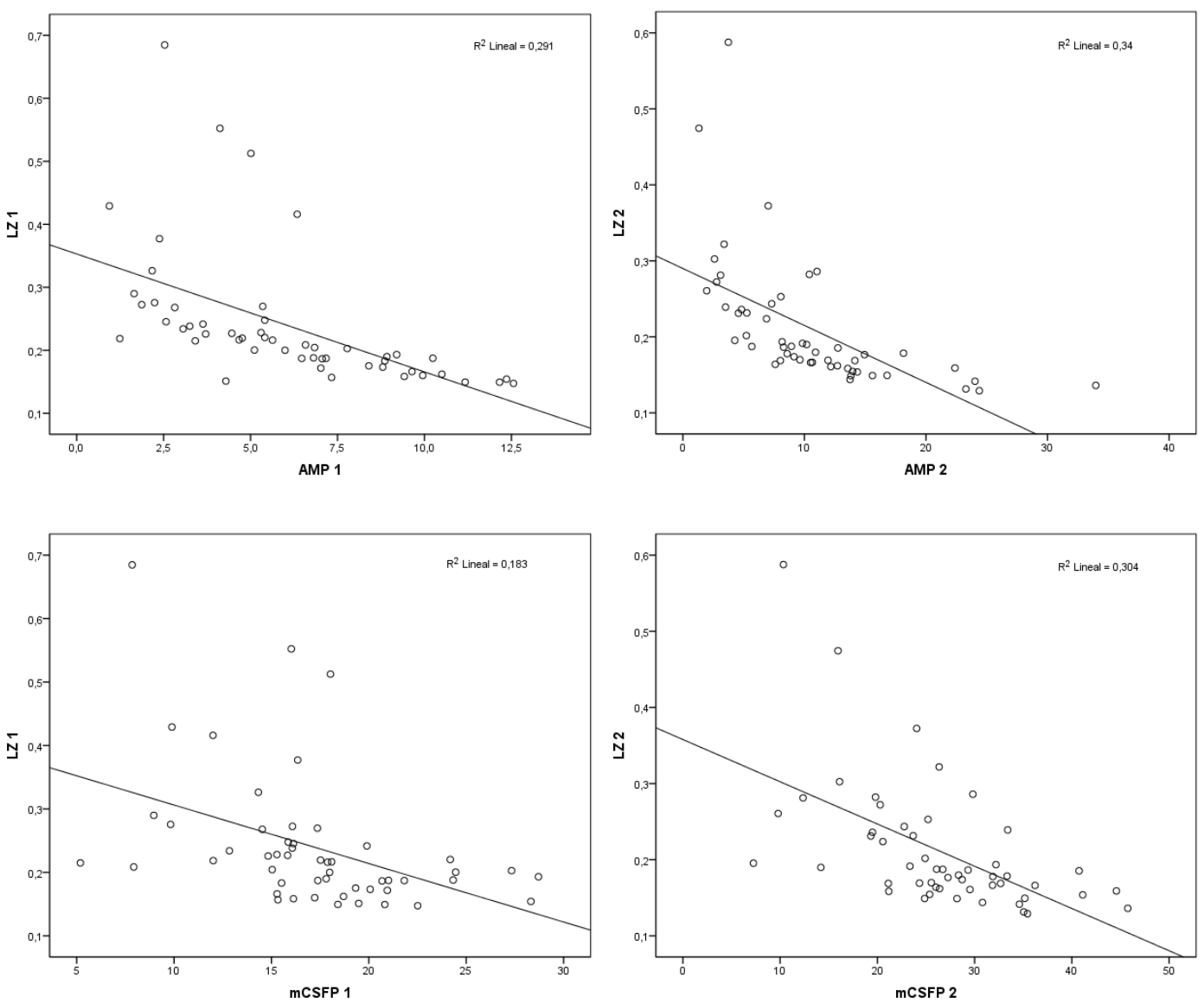

Figure 1. Graphs showing a higher correlation between LZ complexity and pulse amplitude (upper row) than between LZ complexity and mean CSFP (down row) during the infusion period (left column) and during the plateau (right column).

LZ1, LZ complexity during infusion; AMP1, pulse amplitude during the infusion; $m$ CSFP1, mean CSFP during infusion (suffix 2 denotes same parameters during the plateau period). 